\section{Direct Visualisation, Sizing and Counting of Virus and Phage Particles in Liquids}

\section{Bob Carr, and Duncan Griffiths*}

NanoSight Ltd., Salisbury, UK, ${ }^{*}$ NanoSight USA, Costa Mesa, CA duncan.griffiths@nanosight.com

A laser-based, single nano-particle tracking analysis system was described in the March issue of Microscopy Today. The LM series of analyzers from NanoSight allows nanoscale particles such as viruses to be directly and individually visualised in liquids in real time, from which high-resolution particle size distribution profiles can be obtained, figure 1 . The technique is fast, robust, accurate, and low cost representing an attractive alternative or complement to expensive and more complex methods of nano-particle analysis such as dynamic light scattering (photon correlation spectroscopy) or electron microscopy.

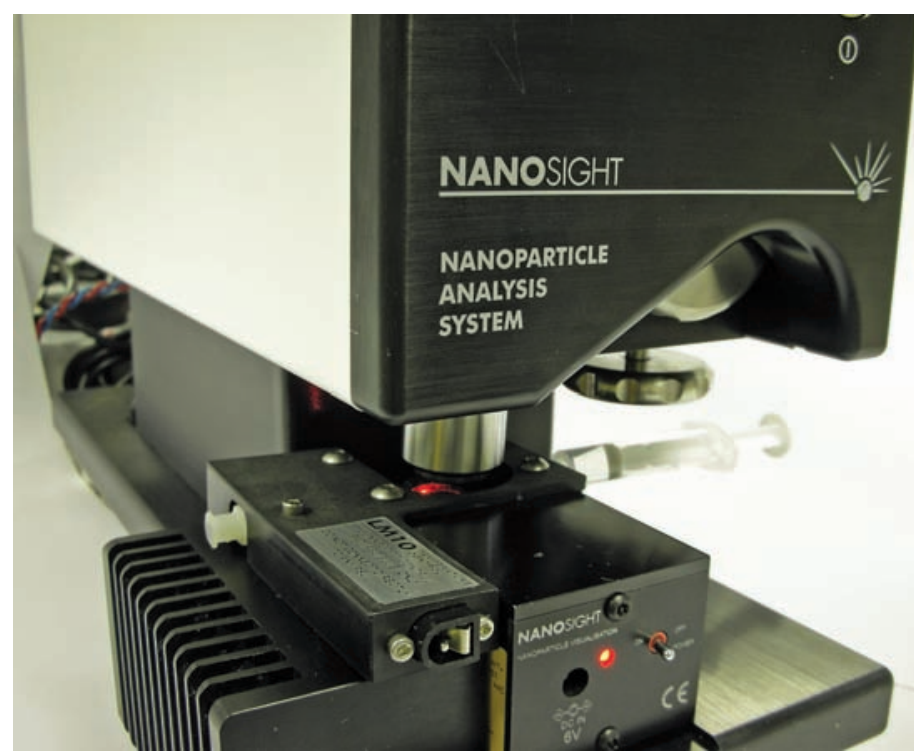

Figure 1: NanoSight LM20 nanoparticle characterization system with temperature stage attached for rigorous control.

By simultaneously and directly measuring the diffusion coefficient of each and every particle, the dedicated Nanoparticle Tracking Analysis (NTA) software suite allows the user to automatically count and size the viruses in a sample. Results are displayed as graphs of size against count of individual particles (or size versus relative brightness), thus overcoming the limitations inherent in other particle analysis systems that generate only mean particle size distribution data with resolution. The NanoSight LM system uses a novel employment of laser light scattering. These instruments visualize and dynamically size populations of suspended particles on an individual basis down to a particle size of approx 20nm, dependent on material. NanoSight directly tracks the Brownian motion of each and every particle separately but simultaneously using a CCD camera and from a high resolution plot of the particle size distribution profile (and changes therein in time). Aggregation and flocculation are immediately apparent. This particle-by-particle approach avoids the ensemble assumptions of dynamic light scattering (DLS) and provides a unique image, going beyond light scattering in assessing polydisperse systems and providing insight into aggregation.
This approach has several advantages. For example, sample pre-treatment is minimal requiring only dilution to $10^{7}-10^{10}$ per $\mathrm{ml}$. Accurate and reproducible analyses can be obtained from video clips of only a few seconds duration and the results allow particle size versus number to be recovered. Given the close to real-time nature of the technique, changes in particle size distribution through aggregation or dissolution can be followed automatically.

A wide range of virus types have been successfully analysed to date (Table 1).

Table 1: examples of virus types studied using NTA

\begin{tabular}{|c|c|}
\hline Adenovirus & Japanese Encephalitis Virus (JEV) \\
\hline Cytomegalovirus (MCMV)) & MULV, murine leukaemia virus \\
\hline Herpes simplex & Porcine papillomavirus \\
\hline Lambda phage & Influenza \\
\hline M13 & Bacculavirus \\
\hline MS2 & Cyanovirus \\
\hline Avian flu simulant & TMV \\
\hline
\end{tabular}

The minimum detectable size measurable depends on the particle type but most viruses and phage types can be seen, sized, and counted. The technique is absolute, requiring no calibration and uniquely allows the user a simple and direct qualitative view of the sample under analysis (perhaps to validate data obtained from other techniques such as PCS) and from which an independent quantitative estimation of sample size, size distribution and

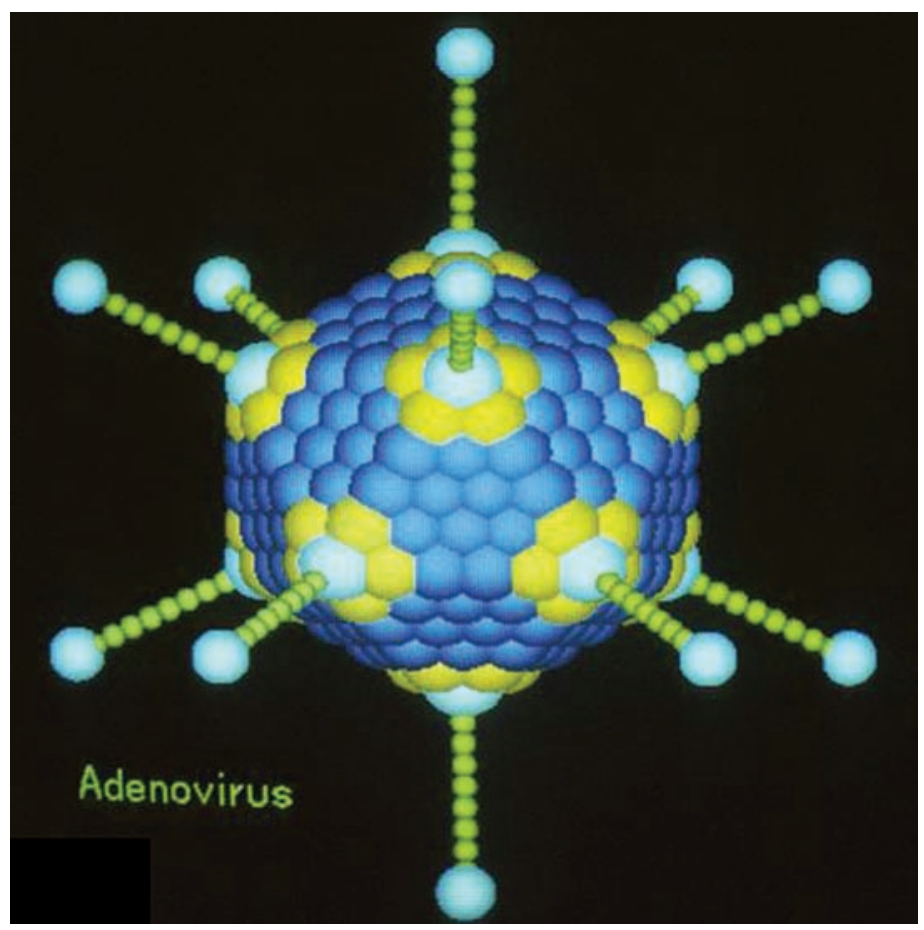

Figure 2: Representation of adenovirus

concentration can be immediately obtained.

The ability to differentiate monodisperse and polydisperse systems clearly in the sub micron region is a key factor for the use of NTA. This is illustrated clearly in studies of adenovirus, 

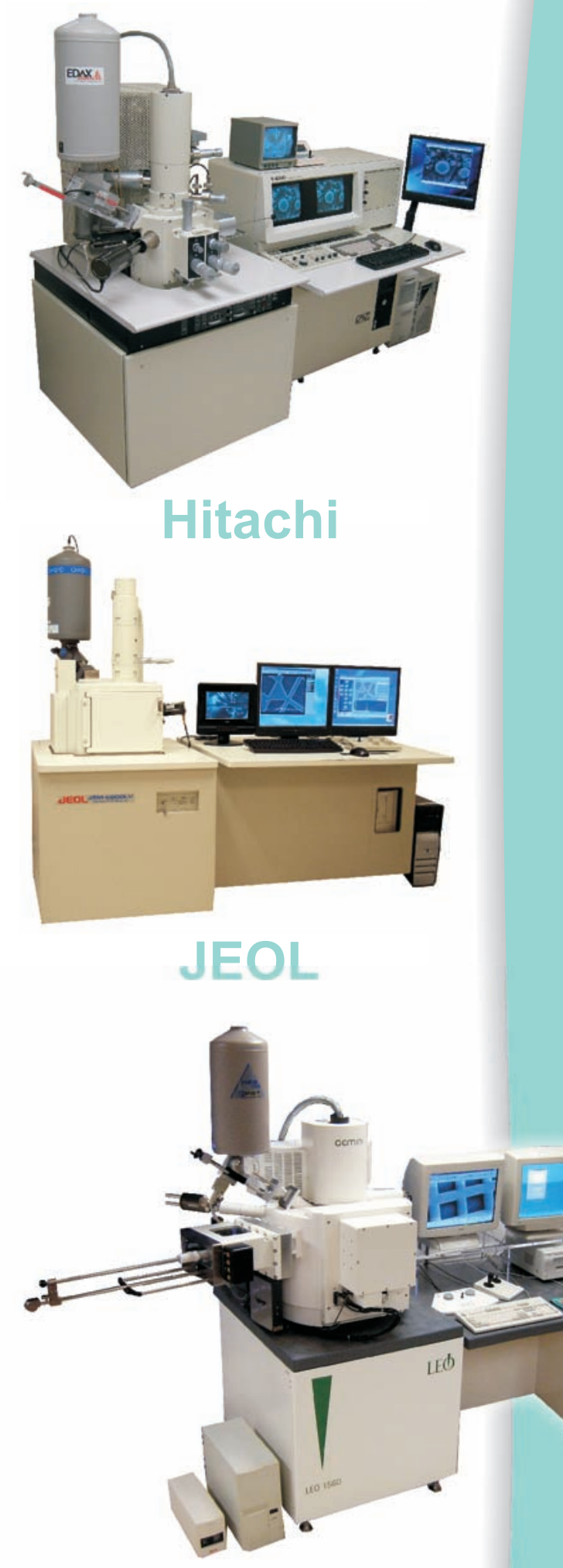

Zeiss \& Others

\section{Why do start-ups, universities, and Fortune 1000 companies rely on SEMTech Solutions for their used SEM needs?}

Its simple. Our pre-owned SEMs are refurbished with the latest technology, and our commitment to service is unrivaled by any used SEM provider.

At SEMTech Solutions, we specialize in upgrading SEMs with new accessories, such as:

- X-Stream Digital Imaging Systems

- IR Chamber Scopes

- New Computer Systems

- EDS \& SDD Upgrades

Our goal is to provide customized SEMs that fit your budget, and offer service packages to meet your long term needs.

\section{Simplified Buying Process}

In-House Demonstrations

Monthly Lease Options

Full Service Options

On-site Installation of SEM/EDS

Basic SEM/EDS Training

Affordable Service Plans 
figure 2. A typical way to display is the use of a particle-scattered intensity versus particle size plot. Figure 3 shows a partially purified influenza-type virus sample in which the vertical axis represents the numbers of virus particles of any given size (particle concentration). It also enables the viewing of aggregated particles of larger size and scattering intensity.

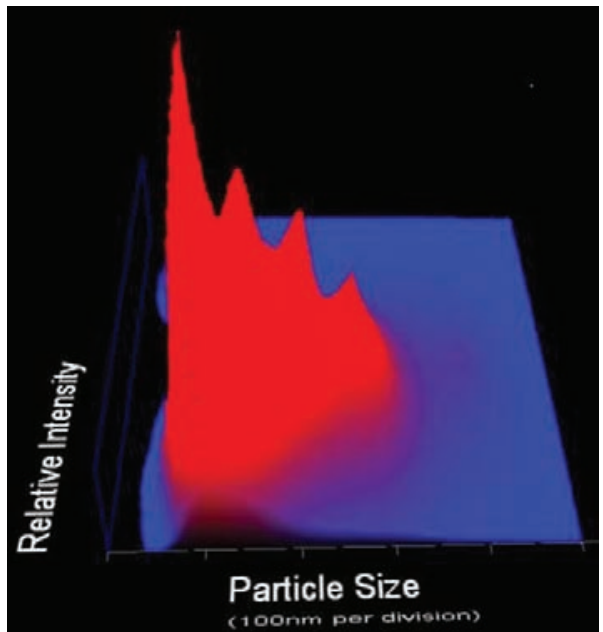

Figure 3: Scattered intensity vs. particle size plot for a partially purified influenza-type virus sample showing aggregation of particles of larger size.

This is in clear contrast with the data shown in figure 4 where a purified suspension of adenovirus shows a clean monodisperse distribution profile with a mode diameter centered at $82 \mathrm{~nm}$, a value that was confirmed using electron microscopy.

\section{Examples of applications for the detection and analysis of viruses:}

\section{Vaccine Production}

Viral vaccine preparations must be proven (validated) to be both stable and to contain known proportions of active ingredients. NTA allows an immediate and direct estimation of product purity and concentration. Similarly, the degree and rate of formation of aggregates in a virus preparation can be simply estimated allowing

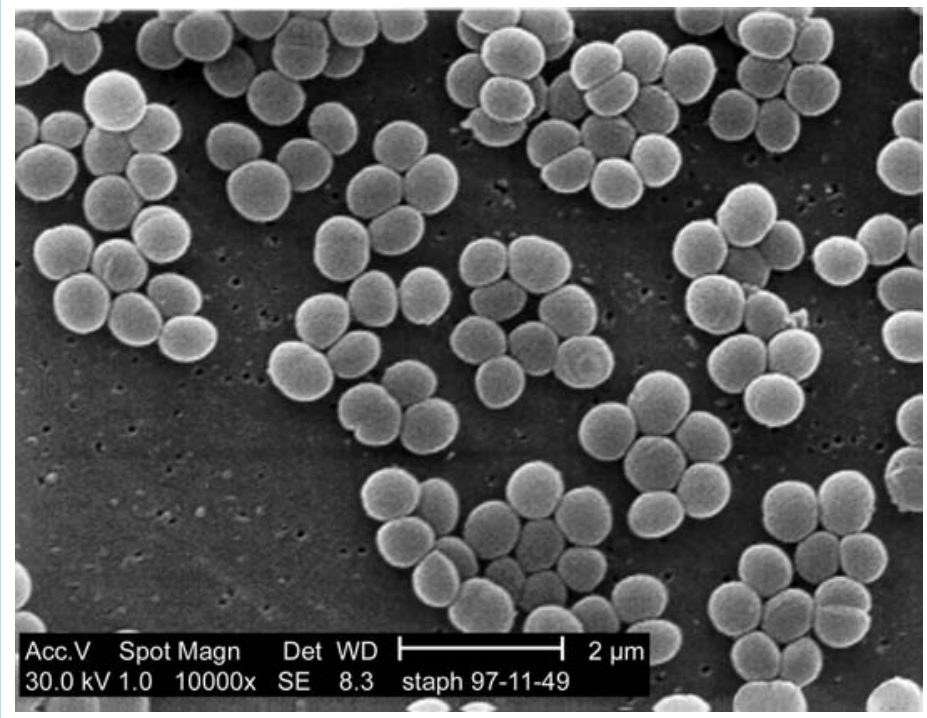

Figure 5: Electron micrograph showing the MRSA virus. the manufacturer to develop improved product manufacturing processes and to optimize product shelf-life.

Because this characterization system allows all particles in the preparation to be visualized and sized, more information about nanoparticle content is made available more quickly to the user than would be available through conventional bio (e.g. plaque) assay. For instance, the presence of larger particles (which can both be sized and counted using NTA) could represent either non-viral cell debris from the cell culture process or aggregates of virus particles containing many individual virions. In either case, such aggregates/contaminants represent a possible problem to the manufacturer.

Bacteriophage-based MRSA Protection - phage therapy

Virus particle detection and counting using Nanoparticle Tracking Analysis is providing essential information for researchers at the University of Strathclyde's Institute of Pharmacy and Biomedical Sciences (IPBS). This team has developed methods to employ naturally-occurring bacteria to combat MRSA, figure 5. MRSA - or Methicillin resistant Staphylococcus aureus - is a variation of the bacterium Staphylococcus aureus, which has developed resistance to most antibiotics, making it difficult to treat and can be potentially deadly. Whilst this "Superbug" can be killed with detergents, detergent dilution and application is often inconsistent and ineffective, hence the bacteriophage route.

Prior to deploying bacteriophages as a dry-coat, to protect high-risk bacterial invasion sites (sutures, instruments and wounds), the cultures need characterisation and their concentration needs assessing. NTA allows the team to view and size viral cultures rapidly, in real time and at low cost. NanoSight provides fast and easy quantitative sample characterisation not possible using other methods and at a much lower cost. Additionally, NanoSight's technology provides a reassuring view of the particle population that supports the counting results.

\section{Virus Clearance Studies}

Virus clearance is assayed (validated) with high titre spikes of viruses (of various types depending on application) that are then used to challenge process steps that need to be qualified as a clearance step. The data must be obtained with pure, non-aggregated virus spike material and this must first be prepared at high titre and stored before use as the spike. NanoSight's methodologies are ideally placed to establish the status of aggregation both before and after storage.

\section{Virus purification}

Finally, the ability of the LM analyzers to rapidly establish the degree of to which a virus preparation contains contaminants or aggregates and to be able to quantify the levels of such, has proved invaluable to process developers interested in optimising purification protocols for virus preparation. 
